\title{
Ellsworth C. (Buster) Alvord, Jr. (1923-2010)
}

Dr. Ellsworth C. (Buster) Alvord, Jr., eminence grise of American neuropathology and longstanding member of the Canadian Association of Neuropathologists, died following a

The genesis of Dr. Alvord's career in neuropathology occurred while a medical student at Cornell University through the formative guidance of Dr. Lewis D. Stevenson. Following his graduation in 1946, Dr. Alvord undertook a curriculum of postgraduate training that included pathology (New York Hospital 1946-1948), neurology (Walter Reed General Hospital 1948-1950), and neuropathology (Armed Forces Institute of Pathology 19501951). Dr. Stevenson's influence pervaded throughout this training and in introducing Dr. Alvord to investigative research on experimental allergic encephalomyelitis as a model for multiple sclerosis.

Spanning 50 years and comprising over half of his 269 publications, Dr. Alvord's work with the experimental allergic encephalomyelitis model represented the dominant theme of his research interests. Integral to the undertaking of these studies was the long

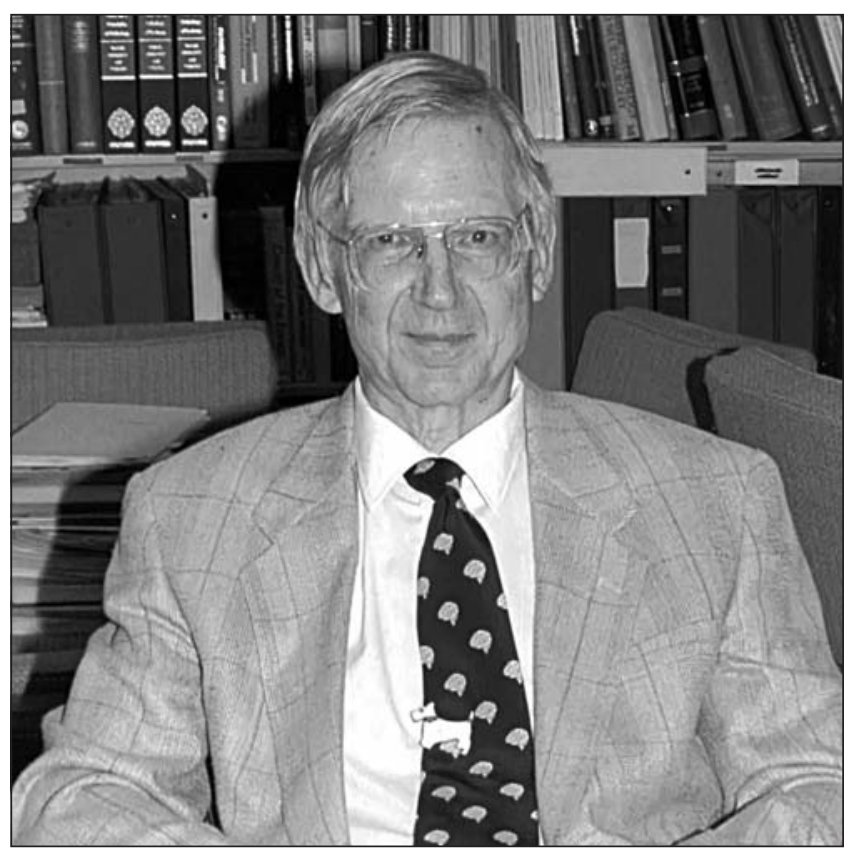
series of strokes on January 19, 2010.

a neuropathology training program instructing more than 45 trainees from the United States, Canada, and other countries. A highlight was the weekly Neuropathology Slide Review Conference. Whether as a trainee (ESJ) or faculty member (HBS), these conferences were equally instructive, challenging and exciting for the tenor of debate and scholarship, and afforded revelatory insight into Dr. Alvord's analytic approach to diagnostic problems.

Late in his career, Dr. Alvord's attention became increasingly focused on predicting the growth of gliomas by mathematical models. With the introduction of MRI and other sophisticated techniques in image analysis, and the forging of collaborative links with colleagues in the field of applied mathematics, it became feasible to calculate the "virtual" growth of high grade gliomas as a means to assess the efficacies of various therapeutic modalities. His earliest paper on the subject was presented in 1987 at the Annual Meeting of the Canadian Association of Neuropathologists, at which occasion he became a member. Thereafter, Dr. Alvord collaborative association formed with Dr. Marion Kies at the National Institute of Neurological Diseases and Blindness, where he had been Chief of the Clinical Neuropathology Section (1953-1955), and with his colleague, Dr. Cheng-Mei Shaw. After moving to the University of Washington in 1960 from Baylor University School of Medicine in Houston (1955-1960), Dr. Alvord had a sustained period of research productivity, making major contributions in identifying the immunogen, developing a model of chronic remitting disease, and devising treatment protocols with monoclonal antibodies. To honor him following retirement in 2002, the Department of Pathology established an annual Alvord Lectureship, among the invited lecturers of which has been Dr. Samuel Ludwin of Queen's University, appropriately speaking on the pathogenesis of multiple sclerosis.

The Neuropathology Laboratory at the University of Washington became a treasure house of clinical material through the provision of a consultation service for medical centers in the Pacific Northwest and beyond. From this repository came many of Dr. Alvord's publications on pediatric neuropathology and congenital malformations of the CNS, culminating with the book, Normal and Abnormal Development of the Human Nervous System. In addition, Dr. Alvord and Dr. Shaw developed regularly presented papers at the meetings, documenting the evolution of mathematical modeling from a concept to an achievable goal applicable to patient care.

Other than neuropathology, there was only one interest of equal importance to Dr. Alvord, that being philanthropy. He and his wife, Nancy, have been repeatedly recognized for the largesse of donations and time bestowed upon various institutions in the greater Seattle area and the University of Washington. However, none will be more lasting than the establishment by the Alvords, their four children and nine grandchildren of the Nancy and Buster Alvord Brain Tumor Center (2009) with the endowment of six Chairs. Buster Alvord bequeathed a notable legacy in neuropathology and, as we celebrate this achievement, so do we the memories of him as an inspiring mentor, generous colleague, and a warm and enriching friend.

Edward Stidworthy Johnson, Edmonton, Alberta Harvey B. Sarnat, Calgary, Alberta

A detailed tribute to Dr. Alvord is forthcoming in the Journal of Child Neurology. 